\title{
Characterization and modeling of dynamic on-body propagation
}

\author{
Bin Zhen ${ }^{\dagger}$, Minseok Kim ${ }^{\ddagger}$, Jun-ichi Takada ${ }^{\ddagger}$ and Ryuji Kohno ${ }^{\dagger}$ \\ National Institute of Information and Communications Technology, 3-4 Hikarino-oka, Yokosuka, 239-0847, Japan ${ }^{\dagger}$ \\ Tokyo Institute of Technology, S6-4, 2-12-1, O-okayama, Meguro-ku, Tokyo 152-8550, Japan *
}

\{zhen.bin@nict.go.jp\}

\begin{abstract}
On-body communication channels are of increasing interest as more and more wireless devices are wearable in medical, military, and personal communication applications. This paper presents an experimental investigation of the dynamic feature of on-body channel due to the movement of human body. Statistical analyses, including level crossing rate, fading duration, and fading interval were conducted. Based on the analyses, a three-state Fritchman model that considers the channel dwelling time is proposed to describe the dynamic characteristic of on-body channel. The channel is classified as unstable good state, constant good state, and bad state. The parameters of the Fritchman model are estimated from the measured data.
\end{abstract}

Index terms: body area networks, on-body propagation, channel characterization.

\section{Introduction}

With the miniaturization of computers, sensors and devices, they can be worn on or attached to the human body for applications in wide-range of fields, including defense, medicine, healthcare and sports [1]. Wireless connections between these devices are preferred for the ease of usage, and the comfort and mobility of the users. One of the most significant features of on-body channel is that the propagation path between two points on the human body is not stationary. Even when standing still, human body is subject to small movements, such as from breathing. During normal activities and sports, the movements become significant. Such movements may also change the operation of antenna.

On-body propagation has been investigated by IEEE 802.15.4 and recently established IEEE wireless body area networks, TG6. The considered frequency bands include industrialscientific-medical (ISM) band, ultra-wideband (UWB) band and wireless medical telemetry (WMTS) band [2-8]. The measurements were conducted in the anechoic chamber, office and hospital. The on-body path-loss can be well described by lognormal distribution, which exceeds the propagation in free space. And the power delay profile can be described based on the well-defined S-V model [9]. Significant channel fading was found when the whole body or part of the body was in movement, such as running and walking $[3,8]$.

The above works considered the on-body channel in term of interference and multipath that combine with the transmitted signal to produce a distorted waveform at receiver. The wireless channel can also be described in a discrete manner using finite-state Markov chain (FSMC). A generalized
Gilbert-Elliott model partitions channel quality into a finite number of intervals, each of which corresponds to one of state of the channel [10-12]. The simplest Gilbert channel has two states, which correspond to total absence of errors and error occurrence with a defined probability, respectively $[10,11]$. The Fritchman model was first introduced in 1967 [12]. For binary channels, Fritchman's framework divides the state space into $k$ good states and $N-k$ bad states according to the signal-to-noise ratio (SNR) at a receiver [13, 14]. The FSMC has been successfully used to model the burst error behavior in flat fading channels.

This paper focuses on modeling the dynamic characteristic of wireless on-body channel induced by continuous human movement. The remainder of the paper is organized as follows. The measurement system and setup in the anechoic chamber are described in section II. Section III presents the statistics for the dynamic fading in the on-body channel. In section IV, a three-state Fritchman model is proposed to capture the burst behavior of the on-body channel. Finally section $\mathrm{V}$ provides concluding remarks.

\section{On-body measurement setup}

As shown in Fig. 1, the measurements were conducted in a radio anechoic chamber where the multi-path from the surrounding objects was negligible. The test subject was a man in his early twenties whose height, chest and waist measurements were $171.5 \mathrm{~cm}, 89.5 \mathrm{~cm}$ and $76.5 \mathrm{~cm}$ respectively. Figure 2 shows the antenna and measurement setup. The transmit antenna $\mathrm{Tx}$ was fixed at a position around navel, and there were 10 receive antenna $R x$ positions. The antenna used (SkyCross ${ }^{\mathrm{TM}}$ SMT-3TO10M-A) was small meander line type that covers wide frequency range between 3.1 to $10 \mathrm{GHz}$. The antenna was mounted $3 \mathrm{~cm}$ above the body surface by formed styrol. The antenna and RF cable were fastened by a belt to minimize their movement during the movements by the subject.

Real-time channel sounder from MEDAV was used for the measurement to capture the dynamic channel behavior [15]. We used it as Single-Input Single-Output (SISO) measurements. The test signal was a periodic 193-tone signal with a center frequency of $4.5 \mathrm{GHz}$ and a bandwidth of 120 $\mathrm{MHz}$, respectively. The frequency domain channel response can be obtained in every $1 \mathrm{~ms}$. The sample rate is sufficient for normal activities [8].

The measurements were conducted one by one at $10 \mathrm{Rx}$ positions. We conducted measurements for three scenarios: 
standing still, walking in place, standing up and sitting down on the chair. The standing still scenario served as reference. The distances between Tx antenna and Rx antennas were given in Table I. Each action was measured for 10s. The subject walked at least 8 steps and stood up-down 5 times in this period. Approximately 10,000 snapshots of channel response were captured by the channel sounder during each measurement.

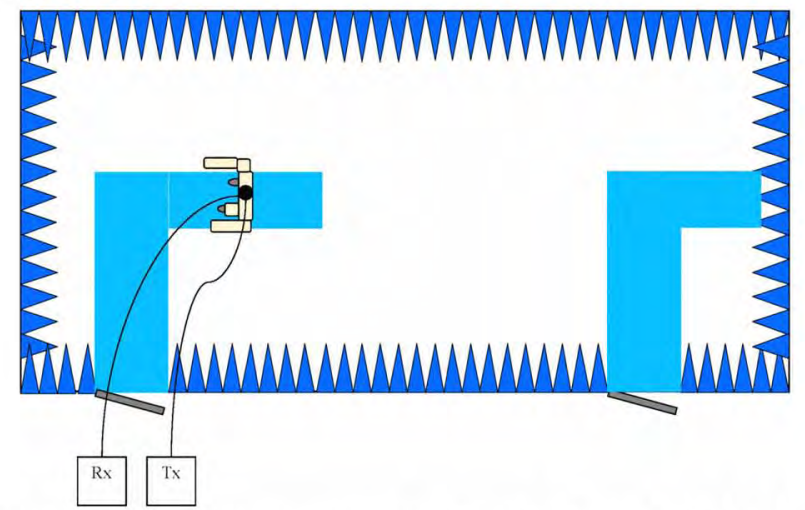

Fig. 1 Layout of the measurement system in the anechoic chamber

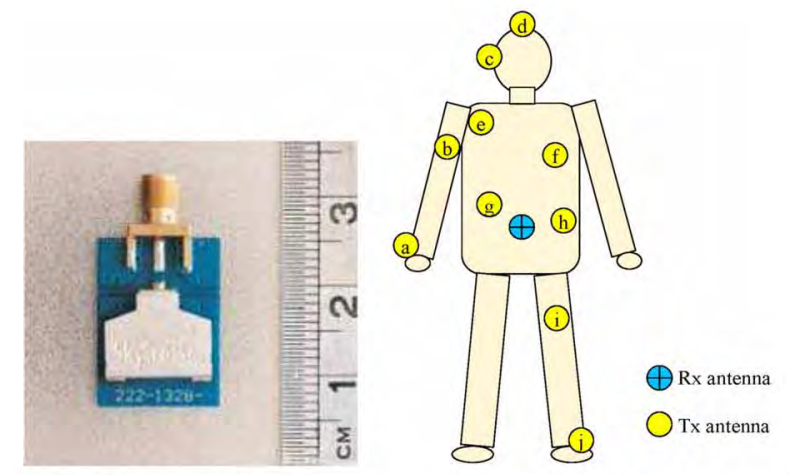

Fig. 2 Antenna and measurement setup locations on the human body (A: right wrist, B: right upper arm, C: left ear, D: head, E: shoulder, F: chest, G: right rib, H: left waist, I: thigh, J: ankle)

\section{Results and analysis}

\section{A. Relative path gain}

For each measurement, we had about 10,000 channel responses in frequency domain. We conducted Fast Fourier Transform operation to convert them into time domain. Then the peak value of the channel impulse responses was considered as the path gain of the measured instant. We then averaged the path gain of the standing still posture to obtain reference path gain of each Rx antenna position. The instant path gain at the position was normalized by the reference path gain to obtain the relative path gain in $\mathrm{dB}$. The relative path gain can remove the impact of distance between the Tx antenna and the Rx antenna at different positions. Also, it can partially de-embed the antenna characteristic from those of propagation path $[2,3]$. We can therefore focus on the dynamic property of the channel.

Figure 3 plots channel response in frequency domain at the right wrist during walking. The plots clearly show that the fading resulting from movement is non-frequency selective in the studied frequency. Figure 4 draws the variation in the relative path gains over time in different scenarios. The channel experiences significant fading that follows the action in a regular base. This is expected as movement causes the separation and orientation of the antenna to change. In some cases, the movement may introduce obstacle between the antennas and significantly change the relative path gain. Even in standing still, irregular and abrupt fading can be found for some points by involuntary movements of the test subject. Table I, which can be found at the end of the paper, shows the statistic parameters of relative path gain, mean value and standard deviation, at each $\mathrm{Rx}$ antenna position.

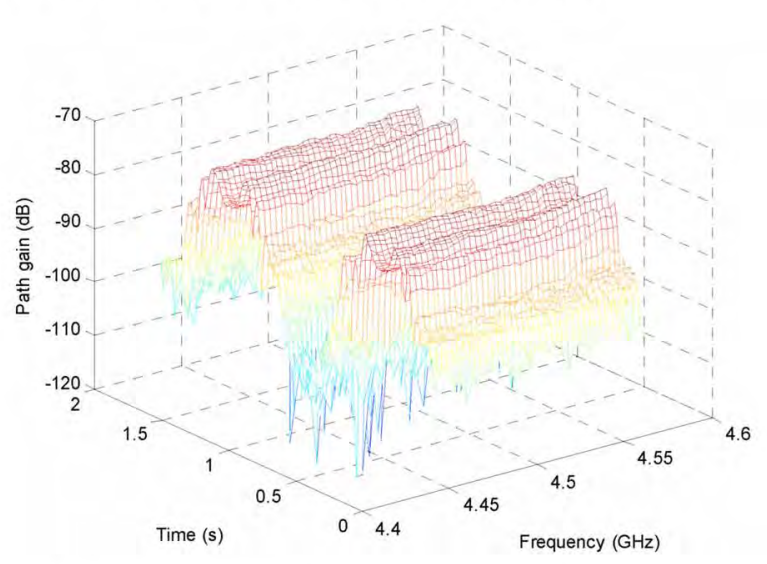

Fig. 3 Channel response in frequency domain at position A (right wrist) during walking

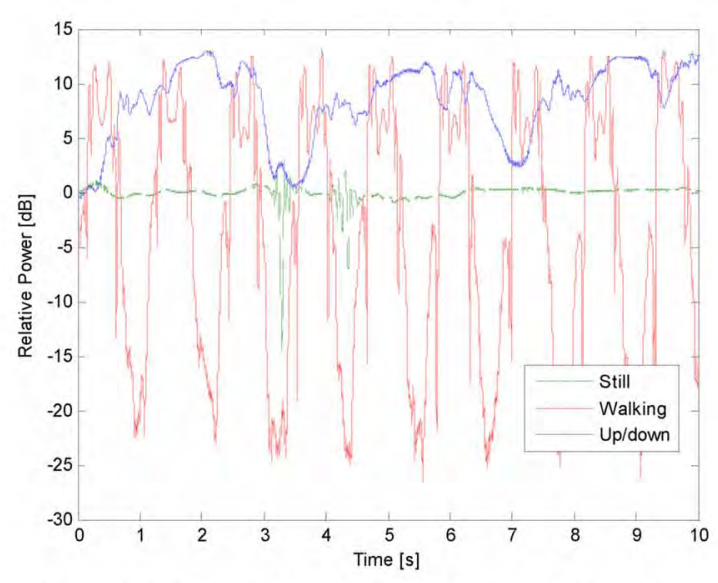

Fig. 4 Relative path gain at position A (right wrist)

\section{B. Statistic analysis}

We can obtain the probability distribution function (PDF) of the relative path gain in the dynamic scenarios. As shown in Fig. 5, the relative path gain can be fitted to a normal distribution, $N(0.1,5.4)$, by using maximum likelihood estimate.

Figure 6 plots the average level crossing rate (LCR), which is the frequency of a signal crosses a threshold in a positive or 
negative going direction, for the two dynamic actions. The channel is good when the relative path gain is over the threshold $\theta$. Otherwise the channel is termed bad. Table I lists the total number of channel switches from good to bad for each action during 10 seconds. As expected, the LCR depends on the mobility of the subject and the antenna position. For example, the LCR was zero at position A during standing up/down; while position B was free of fading during walking. This result is in accordance with the other research about the diversity of on-body links [16].

Given $\theta=-10 \mathrm{~dB}$, we drew the PDF and CDF (cumulative probability function) of fading duration which is the total time for the channel gain is lower than a threshold $\theta$, in Fig. 7, Nearly $90 \%$ of the fading durations are less than $10 \mathrm{~ms}$. This means that the on-body channel is very sensitive to body movements. A small movement, e.g. introducing obstacles between antennas, may have a big impact on the path gain. No bad channel lasts more than $400 \mathrm{~ms}$. The fading duration is related to the velocity of the movement. A slow movement may generate a long fade duration. The measurements was fit by a Gamma distribution, $\Gamma(0.05,0.245)$.

Figure 8 plots the PDF and CDF of the intervals between two adjacent bad channels given the same $\theta=-10 \mathrm{~dB}$. Again, nearly $70 \%$ the interval were within $10 \mathrm{~ms}$. This can be explained by the same reason given above. To our surprise, about $12 \%$ intervals between adjacent bad channels were above $400 \mathrm{~ms}$. This means that the channel may dwell in a good channel for a long time. Again, we fit the measurements (except those larger than $400 \mathrm{~ms})$ by a Gamma distribution, $\Gamma(0.07,4.68)$. Two adjacent bad channels are separated by a good channel. The PDF of the duration of good channels was drawn in Fig. 9. The same as that of bad channels, most good channel lasted less than $10 \mathrm{~ms}$. We found good channels which lasted longer than $400 \mathrm{~ms}$ in both dynamic actions. We calculated the ratio of total duration of good channels to that of bad channels, which was $4.46 \%$. Table I lists the durations of good channels and bad channels at each $\mathrm{Rx}$ antenna position.

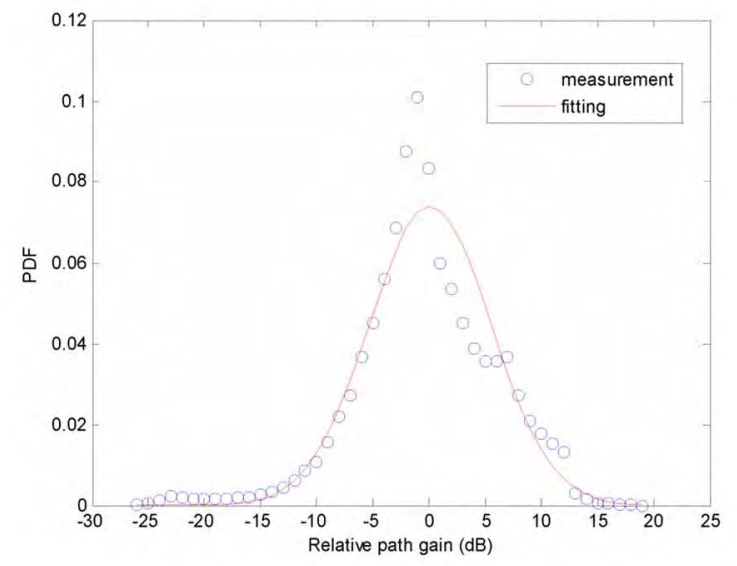

Fig. 5 PDF of relative path gain and its fitting

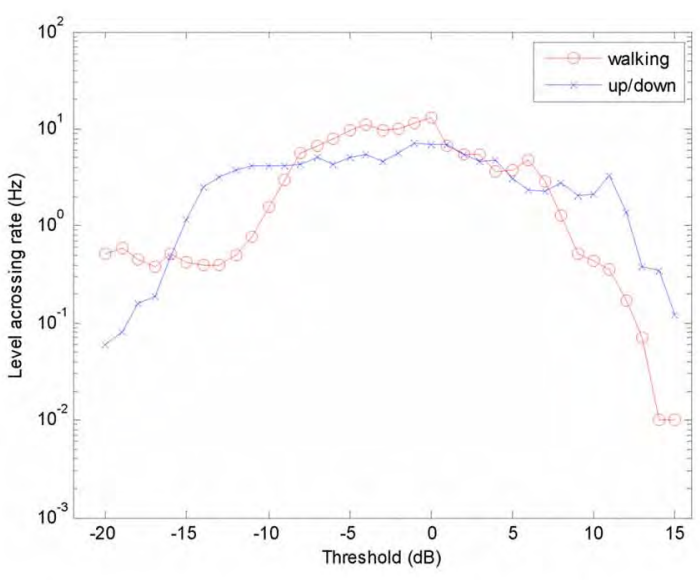

Fig. 6 Average level crossing rate

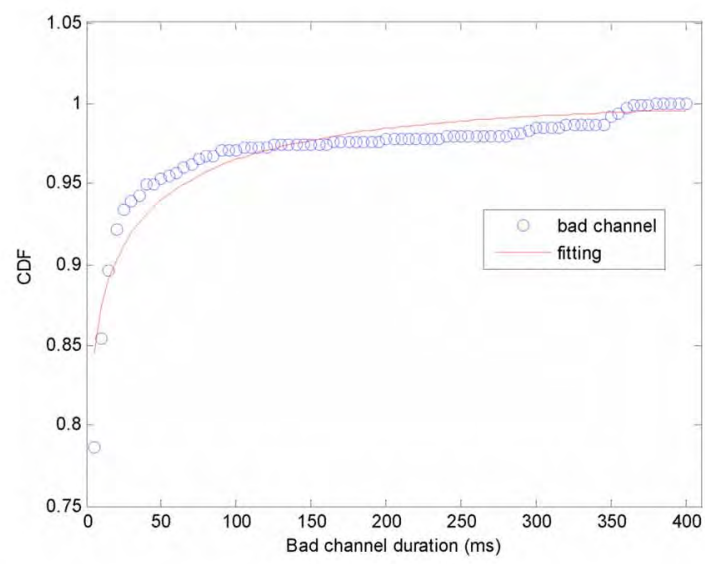

Fig. $7 \mathrm{CDF}$ of the fading duration $(\theta=-10 \mathrm{~dB})$ and its fitting

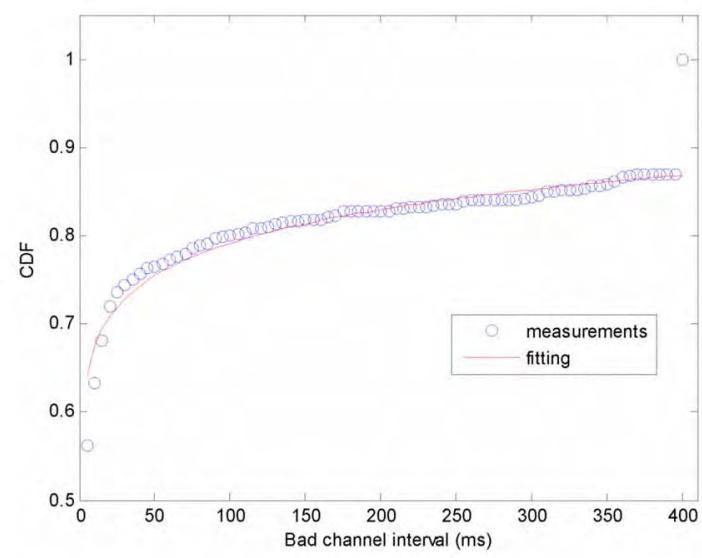

Fig. $8 \mathrm{CDF}$ of the interval between adjacent bad channels and its fitting (-10dB fade threshold) 


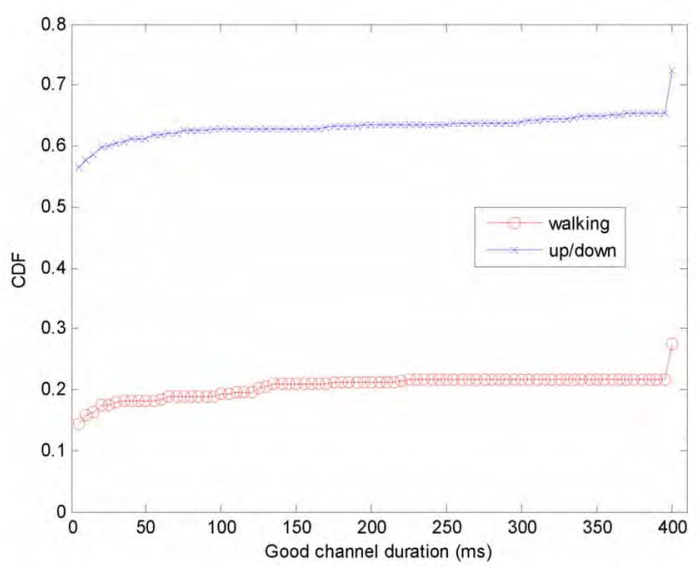

Fig. $9 \mathrm{CDF}$ of the duration of good channel $(\theta=-10 \mathrm{~dB})$

\section{Fritchman channel model}

The measured data shown in Table I, Fig. 8 and Fig. 9 show that the on-body channel dwells in good channel and in bad channel with different patterns. The PDF of the duration of good channel is even not a monotonic function. Therefore, the channel dwelling pattern should be considered in determining the channel state as well as the SNR. Assuming the channel is static within the sample period, Fig. 10 shows a three-state Fritchman model:

- S1: unstable good state, which last less than $400 \mathrm{~ms}$;

- S2: constant good state, which are over $400 \mathrm{~ms}$; and

- S3: bad state.

The good channel is classified into two states. Comparing Fig. 8 and Fig. 9, the PDFs of the unstable good channel and the bad channel are similar. All of them are within $400 \mathrm{~ms}$. The short duration of channel state is dominant. It is therefore not necessary to further classify bad channel into different states. As illustrated in Fig. 10, the state of on-body channel at sample time $t$ is given by

$$
\Pi_{t}=\Pi_{t-1} A \quad t \geq 1,
$$

where $A=\left[\begin{array}{ccc}a_{11} & 0 & a_{13} \\ 0 & a_{22} & a_{23} \\ a_{31} & a_{32} & a_{33}\end{array}\right]$ is the state transit matrix and $\prod o$

is the initial state probability vector. Assume that the good states represent error-free transmissions, and the bad state always produces a transmission error, the error generation matrix takes a very simple form

$$
B=\left[\begin{array}{lll}
1 & 1 & 0 \\
0 & 0 & 1
\end{array}\right] .
$$

We applied the Baum-Welch algorithm to estimate the parameters of the Fritchman model [17]. Table II shows the results for different thresholds.

It is interesting to map the channel fading characteristic on the human body, as shown in Fig. 10. This again confirms that the fading characteristic depends on antenna position and action scenario. Positions $\mathrm{G}$ and $\mathrm{E}$ are totally free of fading. Positions $\mathrm{A}, \mathrm{B}, \mathrm{F}$ and $\mathrm{H}$ suffer from fading during one of the actions. Other positions experience fading during both actions. In the right panel, we classified positions per the features in good channel states. Positions A, C, D and J experience an unstable good state during the dynamic actions, while the other positions remain in the constant good state. This can be partially attributed to the fact that positions $\mathrm{B}, \mathrm{F}, \mathrm{H}$ and $\mathrm{I}$ are all on the trunk of body. They were therefore a short distance from $\mathrm{Rx}$ antenna and had relatively small movement amplitude. It is unexpected to see positions $\mathrm{C}$ and $\mathrm{D}$ that were at head experience severe fading on considering that they were relative static in the dynamic actions. There might be involuntary movements of head during the action scenarios.

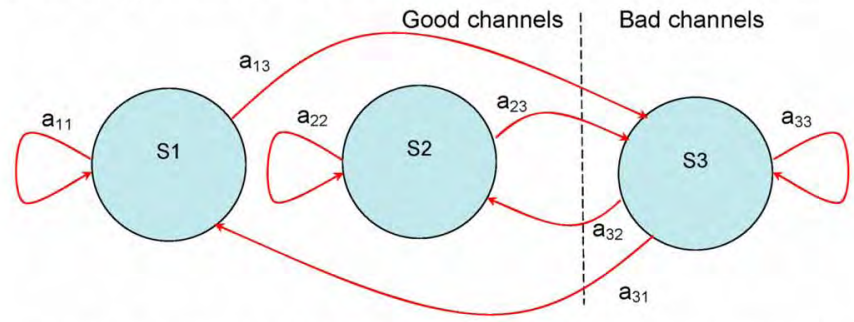

Fig. 10 A 3-state Fritchman model (S1: unstable good state, S2: constant good state, and S3: bad state) for dynamic on-body channels

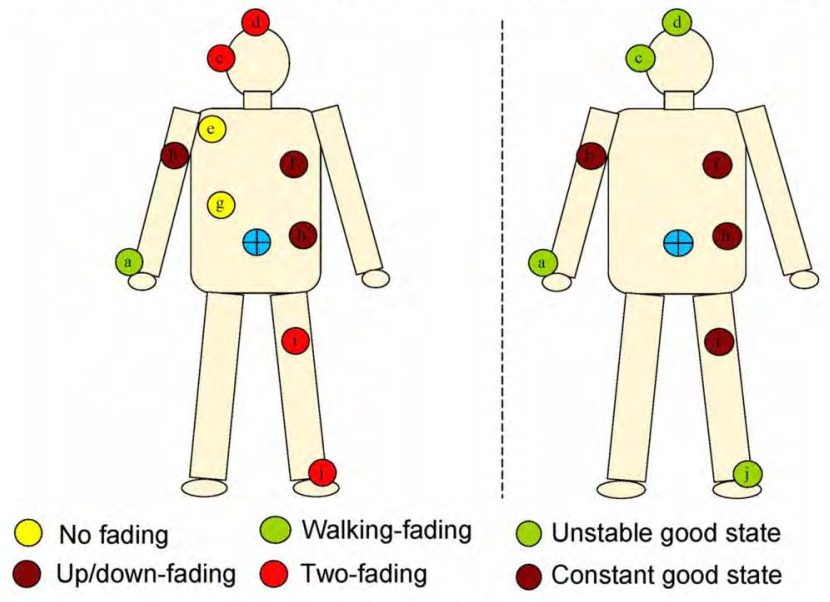

Fig. 11 On-body fading map

\section{Conclusion}

We investigated the dynamic on-body channel at $4.5 \mathrm{GHz}$. The statistical analysis obtained, e.g. LCR and fade duration coincided with research at other frequency bands $[3,4,6,8]$. This indicates that the dynamic feature of the on-body channel is independent of the frequency band. The $4.5 \mathrm{GHz}$ was selected because no channel sounder works at popular $2.4 \mathrm{GHz}$ ISM band. Dynamic channel at $2.4 \mathrm{GHz}$ usually measures the energy at about $10 \mathrm{~ms}$ by using commercial chips. The sample rate is lower than the channel sounder. Another reason is that the UWB is a good candidate for WBAN. The separate distance from body surface to antenna height has a major influence on on-body path loss [19]. We used the same 
separation distance in all scenarios. The antenna height thus has no impact on the fading characteristic.

Traditionally, each FSMC state in the channel model represents a range of received SNR, which in turn determines the error probability of the state [10-15]. Example criterions include bit or packet error rate, packet error distribution, packet throughput, and fade duration [18]. The presented Fritchman model also considered the channel dwell time as another dimension. The $-10 \mathrm{~dB}$ threshold for path loss was selected because it is one of the popular link margins in system design. Because no bad channel lasts longer than $400 \mathrm{~ms}$, it was used as the threshold to divide good channel into two states.

In this paper, we have investigated the dynamic characteristic of the on-body wireless channel in an anechoic chamber. We have measured the channel response at 10 antenna positions in two dynamic scenarios: walking and standing up/down. The statistical analysis for the relative path gain, including LCR, fading duration, and fading interval are presented. The fading feature induced by movement is position and action dependent. Based on the statistical analysis, a three-state Fritchman model has been proposed to describe the time-varying property of onbody fading channel. The channel state is classified into unstable good state, constant good state, and bad state according to the dwelling time in different channel quality. The parameters of the Fritchman model have been estimated from the measured data.

\section{REFERENCES}

[1] D. Cypher, N. Chevrollier, N. Montavont and N. Golmie, "Prevailing over wires in healthcare environments: benefits and challenges," IEEE Communication Magazine, vol. 44, no. 4, p. 56-63, 2006.

[2] K. Y. Yazdandoost, "Channel Model for Body Area Networks (BAN)," IEEE 802.15-08-0033-09, 2008.

[3] P.S. Hall, Y. Hao and Y.I. Nechayev et al, "Antenna and propagation for on-body communication systems," IEEE Antennas and Propagation Magazine, vol. 49, no. 3, p. 41-58, 2007.

[4] S. L. Cotton and W. G. Scanlon, "Characterization and modeling of the indoor radio channel at $868 \mathrm{MHz}$ for a mobile bodyworn wireless personal area network," IEEE Antennas and Wireless Propagation Letters, vol. 6, p. 51-55, 2007.

[5] A. Alomainy, Y. Hao and X. Hu et al, "UWB on-body radio propagation and system modeling for wireless body-centric networks," IEE Proc. Communication, vol. 153, no. 1, p. 107114, 2006.

[6] Z. H. Hu, Y. I. Nechayev and P. S. Hall et al, "Measurements and statistical analysis of on-body channel fading at $2.4 \mathrm{GHz}$," IEEE Antennas and Wireless Propagation Letters, vol. 6, p. 612-616, 2007.

[7] J. Ryckaert, P. De Doncker and R. Meys et al, "Channel model for wireless communication around human body," Electronics Letters, vol. 40, no. 9, p. 543-544, 2004.

[8] D. Miniutti, L. Hanlen, D. Smith and A. Zhang, et al "Dynamic narrowband channel measurements around $2.4 \mathrm{GHz}$ for body area networks," IEEE P802.15-08-0033-00-0006, 2008.

[9] A. Saleh and R. Valenzuela, "A statistical model for indoor multipath propagation," IEEE Communication Mag., vol. 25, pp.5-12, 1987.

[10] E. N. Gilbert, "Capacity of burst-noise channel," Bell Syst. Tech. J., vol. 39, p. 1253-1266, 1960.

[11] E. O. Elliott, "Estimates of error rates for codes on burst-noise channels," Bell Syst. Tech. J., vol. 42, p. 1977-1997, 1963.

[12] B. D. Fritchman, "A binary channel characterization using partitioned Markov chains," IEEE Tran. on Information Theory, vol. 13, no. 2, p. 221-227, 1967.

[13] H. S. Wang and N. Moayeri, "Finite-state Markov channelsa useful model for radio communications channels," IEEE Tran. on Vehicular technology, vol. 44, no. 1, p. 163-171, 1995.

[14] J. Garcia-Frias and P. M. Crespo, "Hidden Markov models for burst error characterization in indoor radio channels," Tran. on Vehicular technology, vol. 46, no. 4, p. 1006-1020, 1997.

[15] Channel Sounder, MEDAV, http://www.channelsounder.de/.

[16] A. A. Serra, P. Nepa, G. Manara and P.S. Hall, "Diversity for body area networks," URSI General Assembly 2008.

[17] L. E. Baum, T. Petrie, G. Soules and N. Weiss, "A maximization techniques occurring in the statistical analysis of probability functions of Markov chain," Annuals of Mathematical Statistics, vol. 41, no. 1, p. 164-171, 1970.

[18] P. Sadeghi, R. A. Kennedy, P. B. Rapajic and R. Shams, "Finite-state Markov modeling of fading channels," IEEE Signal Processing Mag., vol. 25, no. 5, pp. 57-80, 2008.

[19] I. Roelens, S. Van den Bulcke and W. Joseph et al, "Path loss model for wireless narrowband communication above flat phantom," IEE Electronics Letters, vol.42, no.1, p.10-11, 2006. 
Table I Statistical parameters for the dynamic on-body channel (-10 $\mathrm{dB}$ threshold)

\begin{tabular}{|c|c|c|c|c|c|c|c|c|c|}
\hline \multirow[t]{3}{*}{ Positions } & \multirow{3}{*}{$\begin{array}{l}\text { Distance } \\
(\mathrm{mm})\end{array}$} & \multicolumn{4}{|c|}{ Walking } & \multicolumn{4}{|c|}{ Standing up/down } \\
\hline & & \multirow{2}{*}{$\begin{array}{l}\text { Relative path } \\
\text { gain }(\mathrm{dB})\end{array}$} & \multirow{2}{*}{$\begin{array}{l}\text { Level } \\
\text { crossing } \\
\text { (times) }\end{array}$} & \multicolumn{2}{|c|}{ Average duration (ms) } & \multirow{2}{*}{$\begin{array}{l}\text { Relative path } \\
\text { gain }(\mathrm{dB})\end{array}$} & \multirow{2}{*}{$\begin{array}{l}\text { Level } \\
\text { crossing } \\
\text { (times) }\end{array}$} & \multicolumn{2}{|c|}{ Average duration (ms) } \\
\hline & & & & $\begin{array}{l}\text { Good } \\
\text { channels }\end{array}$ & $\begin{array}{l}\text { Bad } \\
\text { channels }\end{array}$ & & & $\begin{array}{l}\text { Good } \\
\text { channels }\end{array}$ & $\begin{array}{l}\text { Bad } \\
\text { channels }\end{array}$ \\
\hline A: right wrist & $440 \sim 525$ & $-3.99 \pm 11.77$ & 31 & 217.03 & 104.97 & $8.45 \pm 3.48$ & 0 & 10,000 & 0 \\
\hline $\begin{array}{l}\text { B: right-upper } \\
\text { arm }\end{array}$ & 360 & $4.45 \pm 2.50$ & 0 & 10,000 & 0 & $6.30 \pm 5.67$ & 16 & 423.33 & 11.88 \\
\hline D: head & 650 & $-3.54 \pm 3.21$ & 37 & 67.64 & 2.78 & $-3.69 \pm 3.21$ & 123 & 62.06 & 10.23 \\
\hline E: shoulder & 310 & $-1.80 \pm 2.05$ & 0 & 10,000 & 0 & $0.22 \pm 4.10$ & 3 & 7 & 1 \\
\hline $\mathrm{F}$ : chest & 230 & $3.64 \pm 2.47$ & 0 & 10,000 & 0 & $5.08 \pm 6.41$ & 17 & 572.56 & 14.12 \\
\hline G: right rib & 183 & $-0.89 \pm 1.40$ & 0 & 10,000 & 0 & $5.70 \pm 3.21$ & 0 & 10,000 & 0 \\
\hline
\end{tabular}

Table II Parameters of the 3-state Fritchman model for different thresholds

\begin{tabular}{|c|c|c|c|c|c|c|c|}
\hline Threshold (dB) & \multicolumn{4}{|c|}{$\boldsymbol{A}$} & \multicolumn{3}{|c|}{$\Pi_{0}$} \\
\hline-6 & $A=$ & {$\left[\begin{array}{c}0.961 \\
0 \\
0.0454\end{array}\right.$} & $\begin{array}{c}0 \\
0.9994 \\
0.00331\end{array}$ & $\left.\begin{array}{c}0.0387 \\
0.000590 \\
0.951\end{array}\right]$ & $\Pi_{0}=[0.14$ & 0.73 & $0.12]$ \\
\hline-10 & $A=$ & {$\left[\begin{array}{c}0.9536 \\
0 \\
0.056\end{array}\right.$} & $\begin{array}{c}0 \\
0.9996 \\
0.0075\end{array}$ & $\begin{array}{c}0.0464 \\
0.000399 \\
0.936\end{array}$ & $\Pi_{0}=[0.054$ & 0.90 & $0.044]$ \\
\hline-14 & $A=$ & $\begin{array}{c}0.922 \\
0 \\
0.0612\end{array}$ & $\begin{array}{c}0 \\
0.9998 \\
0.00722\end{array}$ & $\begin{array}{c}0.0784 \\
0.000183 \\
0.932\end{array}$ & $\Pi_{0}=[0.016$ & 0.96 & $0.02]$ \\
\hline
\end{tabular}

